Peter E. Spronk

Bea Riekerk

José Hofhuis

Johannes H. Rommes

\section{Occurrence of delirium is severely underestimated in the ICU during daily care}

Received: 25 July 2008

Accepted: 16 March 2009

Published online: 7 April 2009

(C) The Author(s) 2009. This article is published with open access at

Springerlink.com

Electronic supplementary material

The online version of this article

(doi:10.1007/s00134-009-1466-8) contains supplementary material, which is available to authorized users.

P. E. Spronk ( ) B Riekerk •

J. Hofhuis - J. H. Rommes

Department of Intensive Care Medicine,

Gelre Hospitals, Location Lukas,

Albert Schweitzerlaan 31,

7334 DZ Apeldoorn, The Netherlands

e-mail: p.spronk@gelre.nl

Tel.: +31-55-5818450

\section{P. E. Spronk}

Department of Intensive Care Medicine, Academic Medical Center, University of

Amsterdam, Amsterdam, The Netherlands

P. E. Spronk

HERMES Critical Care Group,

Amsterdam, The Netherlands
Abstract Objective: Delirium is associated with prolonged intensive care unit (ICU) stay and higher mortality. Therefore, the recognition of delirium is important. We investigated whether intensivists and ICU nurses could clinically identify the presence of delirium in ICU patients during daily care. Methods: All ICU patients in a 3-month period who stayed for more than $48 \mathrm{~h}$ were screened daily for delirium by attending intensivists and ICU nurses. Patients were screened independently for delirium by a trained group of ICU nurses who were not involved in the daily care of the patients under study. The Confusion Assessment Method for the ICU (CAM-ICU) was used as a validated screening instrument for delirium. Values are expressed as median and interquartile range (IQR; P25-P75).

Results: During the study period, 46 patients (30 male, 16 female), median age 73 years $(\mathrm{IQR}=64-80)$, with an ICU stay of 6 days (range 4-11) were evaluated. CAM-ICU scores were obtained during 425 patient days. Considering the CAM-ICU as the reference standard, delirium occurred in $50 \%$ of the patients with a duration of 3 days (range 1-9). Days with delirium were poorly recognized by doctors (sensitivity 28.0\%; specificity $100 \%$ ) and ICU nurses (sensitivity $34.8 \%$; specificity 98.3\%). Recognition did not differ between hypoactive or active status of the patients involved. Conclusion: Delirium is severely under recognized in the ICU by intensivists and ICU nurses in daily care. More attention should be paid to the implementation of a validated delirium-screening instrument during daily ICU care.

Keywords Delirium - Critical care medicine $\cdot$ CAM-ICU $\cdot$ ICU . Recognition

\section{Introduction}

Delirium is an important and frequently occurring complication in intensive care unit (ICU) patients [1-5] associated with a longer ICU stay, longer duration of mechanical ventilation, and higher mortality rates [6]. Consequently, the development of delirium leads to higher costs of care [7]. In view of the strong impact of delirium on clinical outcomes, the early recognition of delirium may result in better outcome if therapy could be started earlier. Nevertheless, the incidence of delirium may be severely underestimated [8], which is an important reason for why specific screening instruments have been developed over time [9]. Both the Confusion Assessment Method for the ICU (CAM-ICU) and the Intensive Care Delirium Screening Checklist (ICDSC) [9] 
may be used as reliable daily diagnostic instruments for the detection of delirium [10]. Recently, the Dutch version of the CAM-ICU was validated as well [11].

In our unit, the nurses and intensivists thought that they were very able to detect delirium in their patients due to the continuous bed-side care in the ICU setting. In addition, no protocol was present in the ICU with respect to the way patients were screened for delirium. Agitated patients were frequently suspected of delirium; in such cases, a geriatrician or psychiatrist was consulted to establish or rule out the presence of delirium. However, the clinical approach to considering delirium seemed to be strongly dependent on the attending physicians and nurses.

We investigated whether ICU nurses and intensivists can clinically identify the presence of delirium in ICU patients during daily care in comparison to the CAM-ICU.

\section{Methods}

Project location

We report here on the clinical recognition of delirium in a university-affiliated teaching hospital in the Netherlands (Gelre Hospitals [GH], Location Lukas, Apeldoorn). In the $\mathrm{GH}$, the ICU is a ten-bed mixed medical-surgical 'closed format' department in which all patients are under the direct care of the ICU team. This team comprises two full-time ICU physicians, five ICU physicians who participate in evening and night shifts, one resident, and 46 full-time equivalent ICU nurses.

\section{Patients}

Only patients with an ICU stay longer than $48 \mathrm{~h}$ were included. Patients with pre-existing neurocognitive dysfunction, documented signs of dementia after psychiatric or geriatric evaluation, language barriers or deafness, an active psychiatric disorder like psychosis, and severe neurological disorders like stroke or meningitis were excluded.

\section{Diagnosing a delirious state}

Over a period of 3 months, both attending ICU physicians and bed-side nurses were asked to score daily every morning between 9:00 a.m. and 10:00 a.m. whether they had the clinical impression that, during that particular day, the patient had signs of delirium, were not delirious, or that the delirious state could not be evaluated, e.g., due to a sedative state. Although they asked the patients involved questions and tried to make contact with them, no structural validated screening instrument for delirium (like the CAM-ICU or the ICDSC) was used, which left them with their 'clinical impression' of whether a delirium was present or not. The level of sedation was routinely evaluated as part of the local sedation protocol using the Richmond Agitation and Sedation Scale (RASS) score [12]. In this protocol, a specific RASS score is recorded in the patient record as the daily goal. A comatose state was defined when the patient had an RASS score $<-3$. Using an objective and validated sedation scale is particularly important when patients develop a hypoactive delirium (in this study, arbitrarily defined as a RASS score from -3 to -1 ), which might be easily missed, whereas a hyperactive delirium (in this study, defined as an RASS score from 2 to 5) may be more obvious. Separately, in the same time window, patients were also evaluated daily by delirium working group research nurses for the presence of delirium using the CAM-ICU. These nurses had been specifically trained in applying the CAM-ICU (see the appendix in the ESM). This evaluation was not disclosed to ICU team members involved in bed-side patient care. In view of previous validation studies of the CAM-ICU in relation to psychiatric evaluation according the DSM-IV criteria $[4,11$, 13-15], the CAM-ICU was considered as the gold standard for the diagnosis of delirium in this study.

\section{Statistical analysis}

The completed data were analyzed using the SPSS version 12.0 statistical software package. Continuous data are expressed as medians with interquartile range (IQR) and categorical data in percentages. Comparisons were made using the Mann-Whitney and Kruskal-Wallis tests for continuous data and the Chi-square test for categorical data. All tests were two-tailed and differences with a $P$-value $<0.05$ were considered as significant.

\section{Results}

During the 3-month study period, 53 patients with an ICU length of stay (LOS-ICU) $>48 \mathrm{~h}$ were eligible for the study. Seven patients were excluded, i.e., due to language barriers $(n=3)$, deafness $(n=2)$, established dementia $(n=1)$, and stroke $(n=1)$, which left 46 patients for the final analysis. The characteristics of those patients are shown in Table 1.

\section{Recognition of delirium}

CAM-ICU scores were obtained during 425 patient days in 46 patients. Scores were positive $(n=89)$, negative $(n=172)$, or non-evaluable because the RASS was $\leq-3$ 
Table 1 Characteristics of patients with $(n=23)$ and without $(n=23)$ delirium during intensive care unit (ICU) stay

\begin{tabular}{|c|c|c|c|c|}
\hline & $\begin{array}{l}\text { Whole group } \\
(n=46)\end{array}$ & $\begin{array}{l}\text { Delirium } \\
(n=23)\end{array}$ & $\begin{array}{l}\text { No delirium } \\
(n=23)\end{array}$ & $P$-value \\
\hline Age (years) & 73 [64-80] & 74 [71-81] & $71[58-80]$ & 0.281 \\
\hline Male (\%) & 65 & 70 & 61 & 0.500 \\
\hline APACHE-II score & 18 [14-20] & $18[15-23]$ & $16[12-20]$ & 0.165 \\
\hline SAPS-II score & $41[35-54]$ & $42[37-55]$ & $40[29-54]$ & 0.222 \\
\hline Mortality $(n(\%))$ & $11(24)$ & $6(26)$ & $5(22)$ & 0.500 \\
\hline Ventilated $(n(\%))$ & $41(89)$ & $21(91)$ & $20(87)$ & 0.500 \\
\hline Days on ventilator & $5[2-8]$ & $6[4-25]$ & $4[1-6]$ & 0.013 \\
\hline LOS-ICU (days) & $6[4-11]$ & $9[6-26]$ & $5[3-8]$ & 0.002 \\
\hline LOS-hospital & 22 [14-37] & $29[21-41]$ & $19[7-30]$ & 0.013 \\
\hline
\end{tabular}

Values are expressed as median and interquartile range $\left[P_{25}-P_{75}\right]$

APACHE Acute Physiology, Age and Chronic Health Evaluation; SAPS Simplified Acute Physiology Score; LOS length of stay

( $n=164)$. Only $28.0 \%$ of the delirious days were picked up by attending intensivists (Table 2). Although nurses performed a little bit better $(34.8 \%)$, their recognition was also poor. Specificity was high for both groups of caregivers $(100 \%$ and $98.3 \%$, respectively). Hence, the occurrence of delirium was severely underestimated, but if delirium was judged to be present on clinical grounds, it was virtually always confirmed by a positive CAM-ICU testing. Sensitivity of the clinical detection of delirium was lower (doctors and nurses: $23.6 \%$ ) during screening moments when patients were in an active state $(n=196)$ when compared to screening moments in patients who were in a hypoactive state (doctors: $28.6 \%$; nurses $42.9 \%$; $n=121$; Table 2). The number of screening moments in patients who were in an agitated state was too low to allow any conclusions to be drawn.

Factors associated with delirium

Considering the CAM-ICU as the gold standard, delirium occurred in $50 \%$ of the patients with a median duration of 3 days (IQR 1-9). Patients with delirium stayed longer on the ventilator (6 days, [4-25]) than those without delirium (4 days, [1-6]; $P<0.001 ;$ Table 1). Consequently, patients with delirium during their ICU stay remained longer in the ICU (9 days, [6-26]) than those without delirium (5 days, [3-8]; $P<0.001$ ). Interestingly, the hospital stay after ICU discharge was also longer in patients who had been delirious (29 days, [21-41]) compared to those who had not been delirious (19 days, [7-30]; $P<0.001)$.

\section{Discussion}

This study demonstrates that both ICU nurses and intensivists have difficulty in diagnosing the presence of delirium in their patients during daily care without a validated, reliable, and easy-to-use diagnostic instrument. Despite a potential Hawthorne effect due to the presence of a 'delirium working group,' the ICU team was insufficiently capable of detecting delirium in critically ill patients, which led to severe underestimation of its prevalence. Only $35 \%$ of the ICU days with delirium were recognized by bed-side nurses, while attending physicians performed comparably. Comparable findings were recently reported by Devlin et al. using the ICDSC, showing that a period of structural training improves the capacity for ICU nurses to diagnose delirium nearly as well as experts [16].

Others have reported that involving ICU physicians in the delirium-screening process by applying tools like the ICDSC [10] and the Neelon and Champagne (NEECHAM) confusion scale [17] improves the diagnostic process. This is important because intensivists play a coordinating role in diagnosing and treating delirium. Indeed, wide variation in the approach towards delirium was reported in a recent Canadian study by Cheung et al. [18]. In particular, the presence or absence of an obvious medical etiology was strongly related to willingness by physicians to call the presence of delirium in that study. The incidence of delirium in this study was comparable to previous reports, although incidences vary between 30 and $80 \%$ [5, 6, 15, 19, 20]. It is intriguing to observe that, even in this small study, a significant impact of delirium was found on ventilator days, length of stay in the ICU, and total hospital stay. This underlines the importance of this entity.

Several limitations to our study should be mentioned. First, we report observations from a single center in the Netherlands. However, the fact that the ICU team composition was constant during the observation period strengthens the data in that the recognition of delirium by critical care providers was rather poor. Also, the morbidity associated with delirium was comparable to previous reports, which makes it unlikely that our results would have been different in a much larger group of patients. Second, this is a small observational study, not designed to evaluate the possible association between delirium and outcome parameters. However, the data are 
Table 2 Comparison between doctors and nurses with respect to the detection of delirium during 425 observation days in 46 ICU patients when the Confusion Assessment Method for the ICU (CAM-ICU) serves as the diagnostic standard

\begin{tabular}{|c|c|c|c|c|c|c|c|c|c|c|}
\hline \multirow{2}{*}{\multicolumn{2}{|c|}{$\begin{array}{l}\text { Clinical judgement } \\
\text { of delirium presence }\end{array}$}} & \multirow{2}{*}{\multicolumn{2}{|c|}{$\begin{array}{l}\text { All observations } \\
(n=425)\end{array}$}} & \multirow{4}{*}{$\begin{array}{l}\text { Comatose state } \\
\text { RASS }-5 /-4 \\
(n=105) \\
\text { NA }\end{array}$} & \multirow{3}{*}{\multicolumn{2}{|c|}{$\begin{array}{l}\frac{\text { Hypoactive state }}{\text { RASS }-3 /-2 /-1} \\
(n=121)\end{array}$}} & \multirow{3}{*}{\multicolumn{2}{|c|}{$\begin{array}{l}\text { Active state } \\
\text { RASS } 0 /+1 \\
(n=196)\end{array}$}} & \multirow{3}{*}{\multicolumn{2}{|c|}{$\begin{array}{l}\text { Hyperactive state } \\
\text { RASS }+2 /+3 /+4 /+5 \\
(n=3)\end{array}$}} \\
\hline & & & & & & & & & & \\
\hline & & \multirow[b]{2}{*}{ Yes } & \multirow[b]{2}{*}{ No } & & & & & & & \\
\hline & & & & & Yes & No & Yes & No & Yes & No \\
\hline \multirow[t]{7}{*}{ Nurse } & CAM: + & 31 & 58 & 0 & 21 & 28 & 9 & 29 & 1 & 1 \\
\hline & CAM: - & 3 & 169 & 2 & 1 & 14 & 2 & 153 & 0 & 0 \\
\hline & CAM: NA & 1 & 163 & 103 & 1 & 56 & 0 & 3 & 0 & 1 \\
\hline & Sensitivity & 0.35 & & - & 0.43 & & 0.24 & & - & \\
\hline & Specificity & 0.98 & & - & 0.93 & & 0.99 & & - & \\
\hline & PPV & 0.91 & & - & 0.95 & & 0.82 & & - & \\
\hline & NPV & 0.74 & & - & 0.33 & & 0.84 & & - & \\
\hline \multirow[t]{7}{*}{ Physician } & CAM: + & 25 & 64 & 0 & 14 & 35 & 9 & 29 & 2 & 0 \\
\hline & CAM: - & 0 & 172 & 2 & 0 & 15 & 0 & 155 & 0 & 0 \\
\hline & CAM: NA & 1 & 163 & 103 & 1 & 56 & 0 & 3 & 0 & 1 \\
\hline & Sensitivity & 0.28 & & - & 0.29 & & 0.24 & & - & \\
\hline & Specificity & 1.00 & & - & 1.00 & & 1.00 & & - & \\
\hline & PPV & 1.00 & & - & 1.00 & & 1.00 & & - & \\
\hline & NPV & 0.73 & & - & 0.30 & & 0.84 & & - & \\
\hline
\end{tabular}

$N P V$ negative predictive value; $P P V$ positive predictive value; $N A$ not applicable

in line with previous findings and illustrate that the patients included represent a common ICU population. Third, one might argue that concurrent sedation may have influenced the results. However, this seems unlikely in view of the finding that the sensitivity of detecting delirium was somewhat higher, but at least not lower, during sedative states than during active states.

In conclusion, in view of the strong impact on patient morbidity and resource consumption [6,7], structural screening for delirium by a validated diagnostic instrument should be a part of routine daily critical care. Further studies should demonstrate whether the implementation of these structural tools results in better patient care and outcome.

Open Access This article is distributed under the terms of the Creative Commons Attribution Noncommercial License which permits any noncommercial use, distribution, and reproduction in any medium, provided the original author(s) and source are credited.

\section{References}

1. Inouye SK (1994) The dilemma of delirium: clinical and research controversies regarding diagnosis and evaluation of delirium in hospitalized elderly medical patients. Am J Med 97:278-288

2. Lipowski ZJ (1987) Delirium (acute confusional states). JAMA 258:17891792

3. Francis J, Kapoor WN (1992) Prognosis after hospital discharge of older medical patients with delirium. J Am Geriatr Soc 40:601-606

4. Pisani MA, Araujo KL, Van Ness PH, Zhang Y, Ely EW, Inouye SK (2006) A research algorithm to improve detection of delirium in the intensive care unit. Crit Care 10:R121

5. Ouimet S, Kavanagh BP, Gottfried SB, Skrobik Y (2007) Incidence, risk factors and consequences of ICU delirium. Intensive Care Med 33:66-73
6. Ely EW, Shintani A, Truman B, Speroff T, Gordon SM, Harrell FE Jr, Inouye SK, Bernard GR, Dittus RS (2004) Delirium as a predictor of mortality in mechanically ventilated patients in the intensive care unit. JAMA 291:17531762

7. Milbrandt EB, Deppen S, Harrison PL, Shintani AK, Speroff T, Stiles RA, Truman B, Bernard GR, Dittus RS, Ely EW (2004) Costs associated with delirium in mechanically ventilated patients. Crit Care Med 32:955-962

8. Pandharipande P, Cotton BA, Shintani A, Thompson J, Costabile S, Truman Pun B, Dittus R, Ely EW (2007) Motoric subtypes of delirium in mechanically ventilated surgical and trauma intensive care unit patients. Intensive Care Med 33:1726-1731
9. Ely EW, Margolin R, Francis J, May L, Truman B, Dittus R, Speroff T, Gautam S, Bernard GR, Inouye SK (2001) Evaluation of delirium in critically ill patients: validation of the Confusion Assessment Method for the Intensive Care Unit (CAM-ICU). Crit Care Med 29:1370-1379

10. Devlin JW, Fong JJ, Schumaker G, O'Connor H, Ruthazer R, Garpestad E (2007) Use of a validated delirium assessment tool improves the ability of physicians to identify delirium in medical intensive care unit patients. Crit Care Med 35:2721-2724

11. Vreeswijk R, Toornvliet A, Honing M, Bakker K, de Man T, Daas G, Spronk PE, de Jonghe JFM, Kalisvaart K (2008) Translation, retranslation and validation of the Dutch Confusion Assessment Method for the Intensive Care Unit. Crit Care 12(Suppl 2):515 
12. Ely EW, Truman B, Shintani A, Thomason JW, Wheeler AP, Gordon S, Francis J, Speroff T, Gautam S, Margolin R, Sessler CN, Dittus RS, Bernard GR (2003) Monitoring sedation status over time in ICU patients: reliability and validity of the Richmond Agitation-Sedation Scale (RASS). JAMA 289:2983-2991

13. Bergeron N, Skrobik Y, Dubois MJ (2002) Delirium in critically ill patients. Crit Care 6:181-182

14. Truman B, Ely EW (2003) Monitoring delirium in critically ill patients. Using the confusion assessment method for the intensive care unit. Crit Care Nurse 23:25-36
15. McNicoll L, Pisani MA, Ely EW, Gifford D, Inouye SK (2005) Detection of delirium in the intensive care unit: comparison of confusion assessment method for the intensive care unit with confusion assessment method ratings. J Am Geriatr Soc 53:495-500

16. Devlin JW, Marquis F, Riker RR, Robbins T, Garpestad E, Fong JJ, Didomenico D, Skrobik Y (2008) Combined didactic and scenario-based education improves the ability of intensive care unit staff to recognize delirium at the bedside. Crit Care 12:R19

17. Immers HE, Schuurmans MJ, van de Bijl JJ (2005) Recognition of delirium in ICU patients: a diagnostic study of the NEECHAM confusion scale in ICU patients. BMC Nurs 4:7
18. Cheung CZ, Alibhai SM, Robinson M, Tomlinson G, Chittock D, Drover J, Skrobik Y (2008) Recognition and labeling of delirium symptoms by intensivists: does it matter? Intensive Care Med 34:437-446

19. Thomason JW, Shintani A, Peterson JF, Pun BT, Jackson JC, Ely EW (2005) Intensive care unit delirium is an independent predictor of longer hospital stay: a prospective analysis of 261 nonventilated patients. Crit Care 9:R375R381

20. Pandharipande P, Jackson J, Ely EW (2005) Delirium: acute cognitive dysfunction in the critically ill. Curr Opin Crit Care 11:360-368 